\begin{tabular}{c} 
International Journal of Engineering \& Technology, 7 (4) (2018) $4602-4606$ \\
International Journal of Engineering \& Technology \\
WPC \\
Website: www.sciencepubco.com/index.php/IJET \\
Research paper \\
\hline
\end{tabular}

\title{
Hybrid DWT-DCT compression algorithm \& a new flipping block with an adaptive RLE method for high medical image compression ratio
}

\author{
S. Rafea $^{1} *$ Dr. N. H. Salman ${ }^{1}$ \\ ${ }^{1}$ Department of Computer Science College of science, University of Baghdad, Baghdad, Iraq \\ *Corresponding author E-mail: samer.rafee93@gmail.com
}

\begin{abstract}
Huge number of medical images are generated and needs for more storage capacity and bandwidth for transferring over the networks. Hybrid DWT-DCT compression algorithm is applied to compress the medical images by exploiting the features of both techniques. Discrete Wavelet Transform (DWT) coding is applied to image YCbCr color model which decompose image bands into four subbands (LL, HL, LH and HH). The LL subband is transformed into low and high frequency components using Discrete Cosine Transform (DCT) to be quantize by scalar quantization that was applied on all image bands, the quantization parameters where reduced by half for the luminance band while it is the same for the chrominance bands to preserve the image quality, the zigzag scan is applied on the quantized coefficients and the output are encoded using DPCM, shift optimizer and shift coding for DC while adaptive RLE, shift optimizer then shift coding applied for AC, the other subbands; LH, HL and HH are compressed using the scalar quantization, Quadtree and shift optimizer then shift coding. In this paper, a new flipping block with an adaptive RLE is proposed and applied for image enhancement. After applying DCT system and scalar quantization, huge number of zeros produced with less number of other values, so an adaptive RLE is used to encode this RUN of zeros which results with more compression.Standard medical images are selected to be used as testing image materials such as CT-Scan, X-Ray, MRI these images are specially used for researches as a testing samples. The results showed high compression ratio with high quality reconstructed images
\end{abstract}

Keywords: An Adaptive RLE (Run Length Encoding); DCT (Discrete Cosine Transform); DWT (Discrete Wavelet Transform)); Flipping Block.

\section{Introduction}

A compression is defined as a process by which the computerized information is modified so that the size required to store the data or the bit-rate required for transmission is reduced. Image compression is one of most important techniques used for efficient storage and transmission of images. It reduces the number of bits without affecting the equality of images and it is used in order to reduce the storage requirement, processing time and duration of transmission [1] [2]. Every day, terabytes of medical images and data are generated through advance imaging techniques such as magnetic resonance imaging (MRI), ultrasonography (US), computed tomography (CT), X-rays and many more recent medical imaging techniques. Storing and transferring these huge voluminous data could be an annoying job. Thereby, to reduce transmission time and storage costs, efficient image compression schemes without degradation of image quality are needed. For this purpose many compression and encoding techniques have been used [2-3]. Figure. 1 shows the block diagram of Compression and decompression Algorithm of DCT [4].

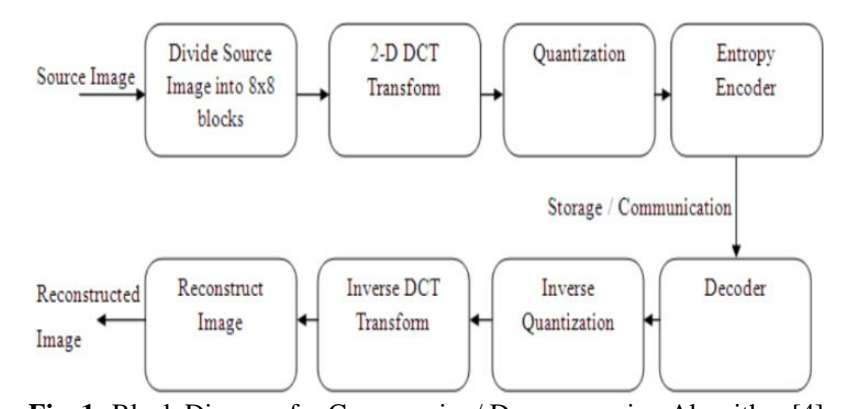

Fig. 1: Block Diagram for Compression/ Decompression Algorithm [4].

\section{Literature survey}

Shaymaa, 2018 [5], proposed an image compression system based on adaptive polynomial transformation. In this work an efficient methods for compression are introduced such still images based of wavelet transform coding and two types of 3D surface representations (Cubic Bezier Interpolation (CBI) and Linear Polynomial approximation). In this work, two coding schemes were developed. The work flow of each scheme consist of two main units; encoding and decoding unit. Image quality is evaluated using fidelity measure (Peak Signal to Noise Ratio (PSNR) and Mean Square Error (MSE)). The system was tested using five tested images, each has different bit depth 8 and 24. The result of conducted tests indicated that the developed system views outstanding compression performance. 
The main objective of the work in [6] was to acquire an effective output of a medical image. This achieved by applying a serial steps starting from image compression and followed by enhance the medical image to achieve an enhanced image output. The detailed analysis of all techniques involved in this process are provided. The quality of images was then evaluated on different performance parameters. The lossy and lossless techniques were used in the process of an image compression. The lossy technique has been done in both DWT and DCT and lossless in both Run Length Encoding (RLE) and process of Block Truncation.

T. Karthikeyan et al, in 2016 [7], focused on compression of images and compares various compression techniques. In this work, four image compression techniques were simulated. The first one is focused on Karhunen-Loève Transforms (KLT), second one was focused on Walsh-Hadamard Transforms (WHT), third technique was based on FFT while the fourth one was proposed Sparse Fast Fourier Transform (SFFT). The experimental results were compared with the various parameters quality applied on many CT scan images of lung cancers. The Proposed algorithm of SFFT technique was given better results like PSNR, SC, MSE and CR compared to other techniques. Also the proposed SFFT gives improved results compared with other techniques in all evaluation measures.

Achinta et al, in 2016 [8], discussed the lossy image compression techniques and reviews of different basic lossy image compression methods. The methods such as JPEG and JPEG2000 are considered for the compression and decompression of images. By considering many images as inputs, it is spotted that MSE PSNR is high and MSE is low in jpeg2000 than jpeg based compression. It was concluded that the overall performance of jpeg2000 is best than jpeg on the basis of compression rates according the mentioned results. In jpeg image need to be "blocked", correlations across block boundaries is not discarded. These results is noticeable and disturbing "blocking artifacts" especially at low bitrates. Wavelets are good to represent the point singularities and could not represent line singularities.

Nitu Rani et al, in 2015 [9], analyzed the comparative performance of DWT DCT transforms based on various parameters. DWT gives high quality and more compression ratio than original image, it adds speckle noise to an image for purpose of improvement in the reconstructed image. So, the DWT technique is useful in a medical data compression. While the DCT compression technique gives less ration of compression but it is computationally effective compared to other techniques.

\section{Proposed methods}

a) Biorthogonal tap 9/7 wavelet transform coding

DWT is used in medical imaging where image declination is not used to improve and process signals, in fields such as medical imaging where image degradation is not tolerated they are of particular use. They can be used to remove noise in an image, for example if it is of very fine scales, wavelets can be used to cut out this fine scale, effectively removing the noise. The goal of wavelet -transform encoding is to take advantage of redundancy in the transformed image and obtain a good reconstruction upon decompression transform of it [10]. Figure 2 and 3 shows the process of applying DWT Tap 9/7 for MRI and X-Ray images.

b) Discrete wavelet transform coding

In this work, after image color transformation process, the Discrete Wavelet Transform is applied to each band of the transformed color space components $\mathrm{Y}, \mathrm{Cb}$ and $\mathrm{Cr}$ separately, where the DWT represents image data into two sets of coefficients; High pass coefficients (detailed part of an image) and low pass coefficients. Each band of color space is decomposed into four subbands (LL, LH, HL and $\mathrm{HH}$ ), each subband carry a specific part of image information as shown in figure 2. With DWT, mathematical functions is used to map an image information to an alternate representation in such way that most of an image energy is concentrated into low frequency bands LL. All subbands are discarded except the low fre- quency band LL which transformed using the DCT transform coding, while the other subbands; $\mathrm{LH}, \mathrm{HL}$ and $\mathrm{HH}$ are compressed using the scalar quantization, Quadtree and shift optimizer. Equations 1-6 below are applied to accomplish DWT transformation.

$\operatorname{Re}(\mathrm{n})=\operatorname{Re}(\mathrm{n})+\mathrm{a}[\operatorname{Re}(\mathrm{n}-1)+\operatorname{Re}(\mathrm{n}+$

1)] $\forall$ odd $n$ in the range eq.

$\operatorname{Re}(\mathrm{n})=\operatorname{Re}(\mathrm{n})+\mathrm{b}[\operatorname{Re}(\mathrm{n}-1)+\operatorname{Re}(\mathrm{n}+$

1)] $\forall$ even $n$ in the range eq.

$\operatorname{Re}(\mathrm{n})=\operatorname{Re}(\mathrm{n})+\mathrm{c}[\operatorname{Re}(\mathrm{n}-1)+\operatorname{Re}(\mathrm{n}+$

1)] $\forall$ odd $n$ in the range eq.

$\operatorname{Re}(\mathrm{n})=\operatorname{Re}(\mathrm{n})+\mathrm{d}[\operatorname{Re}(\mathrm{n}-1)+\operatorname{Re}(\mathrm{n}+$

1)] $\forall$ even $n$ in the range eq.

$\operatorname{Re} 1(\mathrm{n})=\frac{\operatorname{Re}(\mathrm{n})}{\mathrm{e}} \forall$ even $\mathrm{n}$ in the range eq.

$\operatorname{Re} 1(\mathrm{n})=\operatorname{Re}(\mathrm{n}) \times \mathrm{e} \forall$ odd $\mathrm{n}$ in the range eq

Figures 2 and 3 shows applying DWT TAP9/7 to MRI and the process steps to X-Ray images respectively

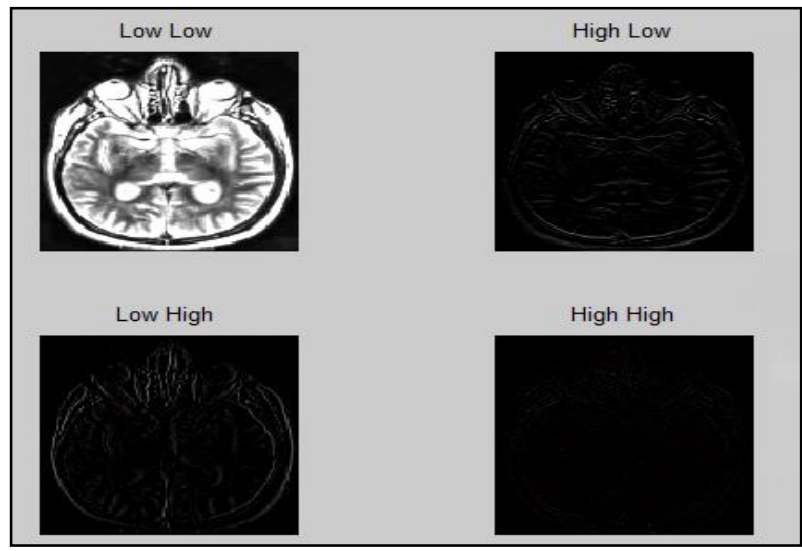

Fig. 2: Applying DWT TAP9/7 to MRI--Brain Image.

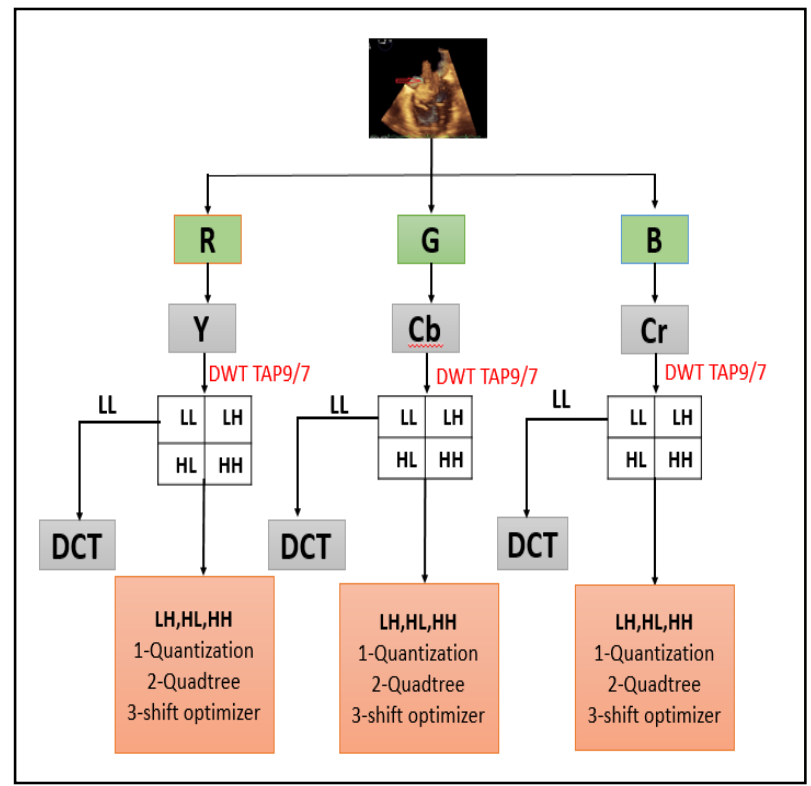

Fig. 3: Process of Applying DWT TAP9/7 to X-Ray.

c) Discrete Cosine Transform (DCT)

Discrete Cosine Transform (DCT) is an image compression technique used for lossy image compression more than any other techniques. DCT demonstrate an image as a sum of sinusoids of varying frequencies and magnitudes. For typical image, the DCT has the property that most of the visually considerable information about 
the image is concentrated in just a few coefficients of the DCT. Compression using Discrete Cosine Transform (DCT) divides up the image into 8 by 8 pixel blocks and then calculates the discrete cosine transform (DCT) of each block.

In this step, the Discrete Cosine Transform Coding is applied to the low frequency subband (LL) [4], [11] [12]. The LL subband is partitioned into $8 \times 8$ blocks of images. Each $8 \times 8$ data block is a subject of discrete cosine transform (DCT). DCT is applied for each block from left to right and top to bottom. DCT Separate image data into two sub-bands of varying importance [13] [14]. Figure 4 shows the process steps of applying DCT to MRI-Brain image.

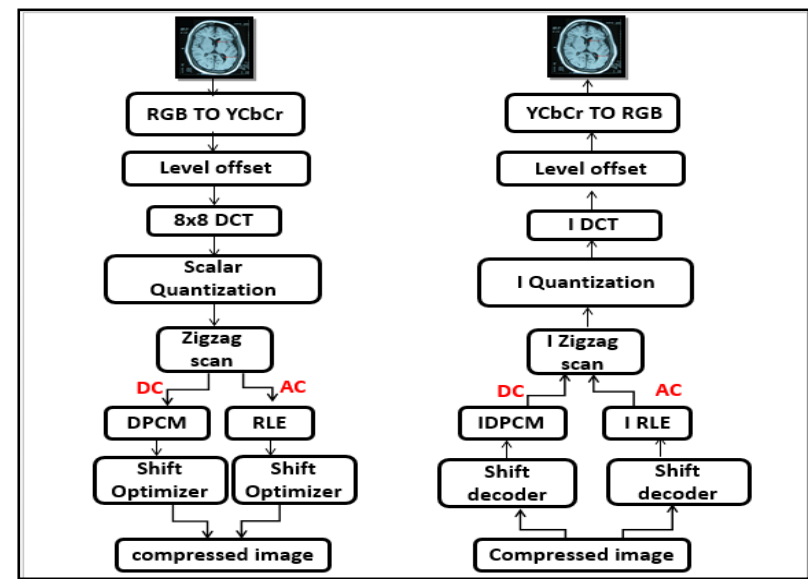

Fig. 4: Process of Applying DCT to MRI-Brain Image.

The output of DCT transforming is a two dimensional matrix resides the DCT coefficients which contains integers which are arranged in a way that a valuable and important information (low frequency coefficients) in the upper left corner of the 2D-matrix and the bottom right corner holds the less significant coefficients (high frequency coefficients) as shown in figure 5. The upper left corner position $(0,0)$ in each $8 \times 8$ block represents the average of the other values in the $2 \mathrm{D}$-matrix, which are the AC coefficients. Equation 7 is applied to obtain 2D DCT transformed matrix. The result of DCT transformation is quantized using an adaptive scalar quantization.

$\mathrm{f}(\mathrm{u}, \mathrm{v})=$

$\frac{2}{\mathrm{~N}} \mathrm{C}(\mathrm{u}) \mathrm{C}(\mathrm{v}) \sum_{\mathrm{x}=0}^{\mathrm{N}-1} \sum_{\mathrm{y}=0}^{\mathrm{N}-1} \mathrm{f}(\mathrm{x}, \mathrm{y}) \cos \left[\frac{\pi(2 \mathrm{x}+1) \mathrm{u}}{2 \mathrm{~N}}\right] \cos \left[\frac{\pi(2 \mathrm{y}+1) \mathrm{v}}{2 \mathrm{~N}}\right]$

For $\mathrm{u}=0, \mathrm{~N}-1$ and $\mathrm{v}=0, \ldots, \mathrm{N}-1$ eq.

Where $\mathrm{N}=8$ and $\mathrm{C}(\mathrm{k})=\left\{\begin{array}{l}\frac{1}{\sqrt{2}}, \text { fork }=0 \\ 1, \text { otherwise }\end{array}\right.$
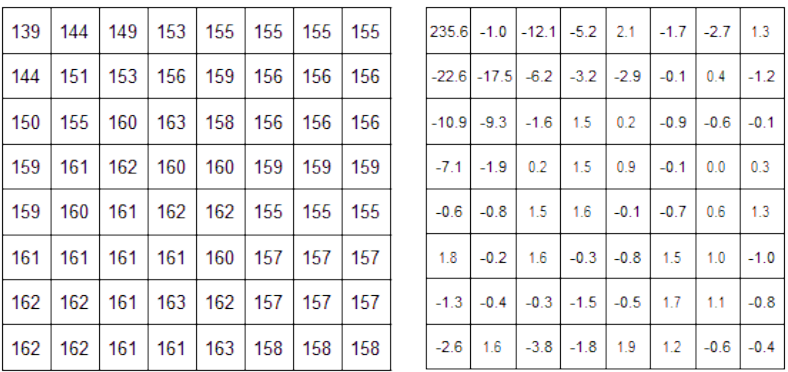

Fig. 5: 8 X 8 Image Block after Applying DCT.

\section{d) Hybrid DCT-DWT}

The benefits of applying a hybrid DCT-DWT is to exploit the properties of both techniques. By giving consideration to the type of application, original image of size $256 \times 256$ or any resolution, provided divisible by 32 , is first divided into blocks of $\mathrm{N} \times \mathrm{N}$. Then each block is decomposed using two dimensional 2-D DWT. The LL subband component is transformed using 8-point DCT. To achieve a higher compression, majority of high coefficients can be ignored. To get more compression a scalar quantization is performed. In this step, many of the higher frequency components are rounded to zero. The quantized coefficients are further scaled using the scaling factor (SF). Then the image reconstructed by the subsequence inverse procedure. During inverse DWT, zero values are lined in place of detailed coefficients [10]. Figure 6 shows the process steps of applying hybrid DCT-DWT to MRI-Brain Image.

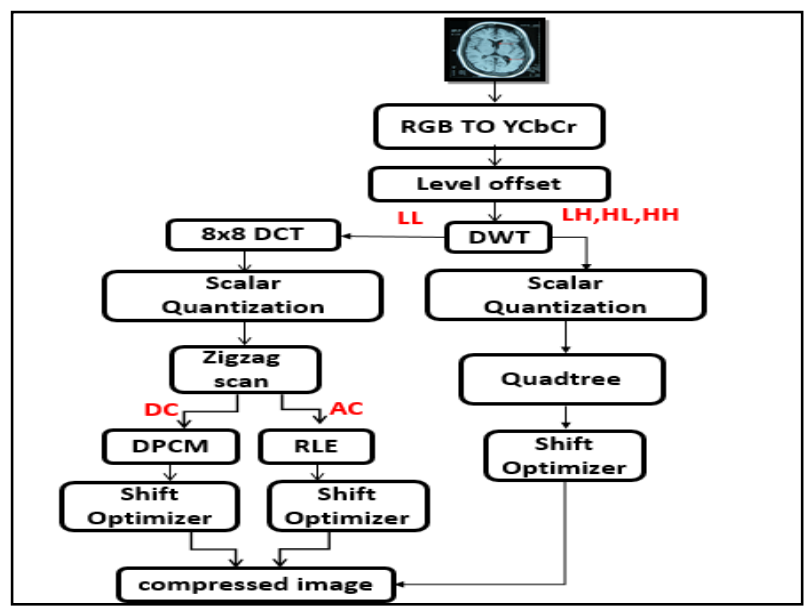

Fig. 6: Process of Applying Hybrid DCT-DWT to MRI-Brain Image.

e) New Flipping Block Technique

After applying quantization and Zig-Zag scan, one dimensional vector is obtained with many consequence of zeros. In this paper, a new flipping block method is applied for AC coefficients. A new flipping block is a rearrangement method used to represents the zeros in AC coefficients vectors as a RUN of zeros to be compressed by adaptive RLEs. It's idea is based on representing each pairs of vectors in such a way that the even vector is inverted from right to left, so that, the zeroes of the pair be as a sequence (RUN) of zeroes. After the Zigzag process, the end of each vector results with a sequence of zeroes. Flipping Block is used to reduce the RUN of zeroes to be written once instead of twice. By using this method, more compression will be gained when applying an adaptive RLE because the flipping method represents the zeros in vector as a RUN and more number of zeros will reduced. Figure (7) shows an example of applying new flipping block.

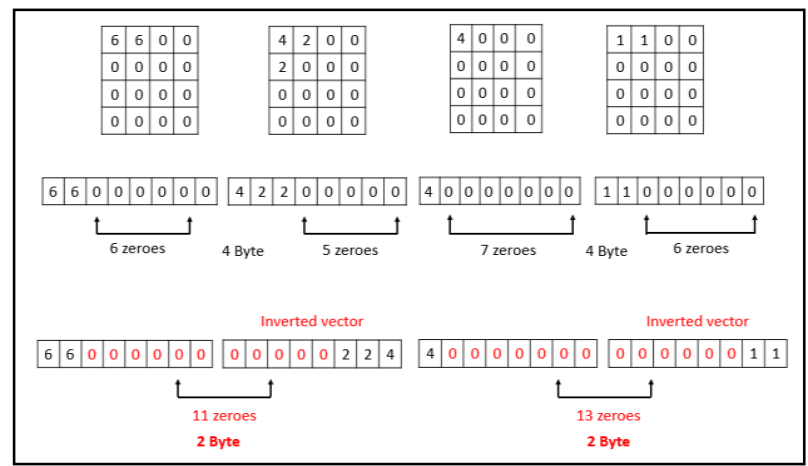

Fig. 7: An Example of Applying New Flipping Code.

\section{f) Adaptive Run Length Encoding}

RLE is a simple method of compression used for data size reduction by replacing the consecutive repeating occurrences of a symbol by one occurrence of the symbol followed by the number of occurrences. RLE is working more efficient if the data to be compress is in form of 1 and 0 . RLE is used to compress the repeated values of AC coefficients. Each repeated values are called RUN and represented by a pair of values, the first value indicates the number of repeated value in the RUN, while the second value indicates the begin value of the RUN. In this thesis, an image enhancement method is proposed as an adaptive RLE is applied to the vector of AC coefficients results from applying of a new flipping block 
method. The new RLE is based on removing the RUN of zeros in vector and represents the vector by place the number of pixels before the RUN of zeros and place the pixels then the number of zeros in the RUN. RLE is used because after DCT is applied, huge number of zeros produced with less number of other values, an adaptive RLE is used to encode this RUN of zeros.

Figure 8 shows the difference between applying an adaptive RLE and traditional RLE method. When applying traditional RLE, the vector of coefficients (Vector A) is represented by pairs denotes the number of repeated values in the RUN and the beginning of the RUN. In an adaptive RLE, the output (vector B) is represented by place the number of pixels before the RUN of zeros and place the pixels then the number of zeros in the RUN and repeat this process for the rest of vector. By applying an adaptive RLE, the number of pixels is reduced from (33) to (13) compared to the traditional RLE which reduce the number of pixels to (17). Therefore, more compression is gained when applying the new RLE method compared to the traditional RLE.

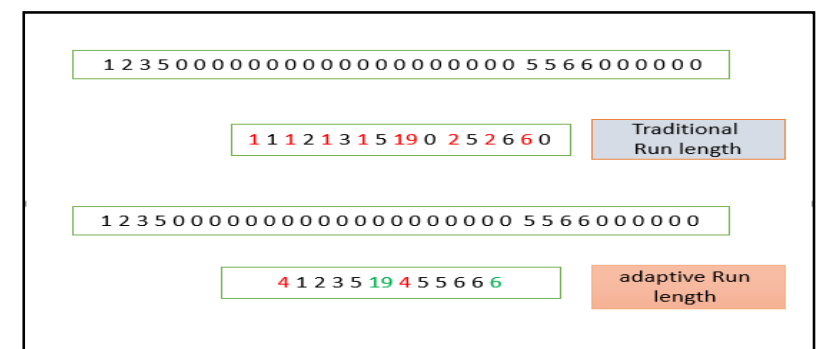

Fig. 8: Difference between Applying an Adaptive RLE and Traditional RLE.

g) Comparative Analysis of the results

The proposed compression system is tested using medical images (such as; X-Ray, CT-Scan, MRI-Brain, MRI-Knee) with characteristics of three bands RGB ,Bitmap image file type, size of 256x256 and 24 bitdepth. These images are used as test samples to evaluate the performance and quality of the designed image compression system. The selected images are standard medical images and used specially for researches. Table (1) shows the final results for CR, PSNR and MSE for (X-Ray, MRI-BRAIN, CT-SCAN and MRIKnee) test images when the DCT and Hybrid DCT-DWT is applied before and after enhance coding.

Table 1: Final Results with Default Value

\begin{tabular}{lccc}
\multicolumn{4}{c}{ Table 1: Final Results with Default Value } \\
\hline image & CR & PSNR & MSE \\
\hline $\begin{array}{l}\text { X-Ray } \\
\text { With DCT only }\end{array}$ & 28.58 & 30.66 & 55.73 \\
$\begin{array}{l}\text { X-ray } \\
\text { With(DCT-DWT) Before enhancement }\end{array}$ & 34.64 & 30.12 & 63.16 \\
$\begin{array}{l}\text { X-Ray } \\
\text { With(DCT-DWT) after enhance }\end{array}$ & 43.08 & 30.12 & 63.16 \\
$\begin{array}{l}\text { MRI-BRAIN } \\
\text { With DCT only }\end{array}$ & 16.10 & 30.80 & 53.87 \\
$\begin{array}{l}\text { MRI-Brain } \\
\text { With(DCT-DWT) Before enhancement }\end{array}$ & 21.31 & 30.07 & 63.84 \\
$\begin{array}{l}\text { MRI-Brain } \\
\text { With(DCT-DWT) after enhancement }\end{array}$ & 27.45 & 30.07 & 63.84 \\
$\begin{array}{l}\text { CT-Scan } \\
\text { With DCT only }\end{array}$ & 30.17 & 30.61 & 56.44 \\
$\begin{array}{l}\text { CT-Scan } \\
\text { With(DCT-DWT) Before enhancement }\end{array}$ & 36.84 & 30.71 & 55.13 \\
$\begin{array}{l}\text { CT-Scan } \\
\text { With(DCT-DWT) after enhancement }\end{array}$ & 46.09 & 30.71 & 55.13 \\
\hline
\end{tabular}

\section{Image reconstruction}

After test images are compressed using the enhanced image compression system mentioned above, the compressed image are reconstructed by the decompression unit of the proposed system. Figures 9, 10, 11 and 12 show the samples of the reconstructed MRI-Brain, X-Ray, CT-Scan and MRI-Knee images respectively where the
PSNR value is varied. Also they show the performance test values (CR, PSNR and MSE) for each reconstructed image.

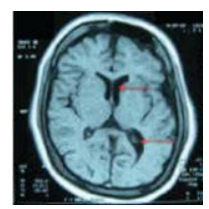

$\mathrm{CR}=27.50$

$\mathrm{PSNR}=30.05$

MSE $=64.18$

Fig. 9: Samples

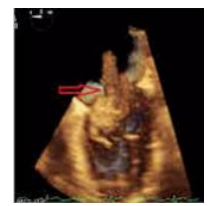

$\mathrm{CR}=43.08$

$\mathrm{PSNR}=30.12$ $\mathrm{MSE}=63.16$

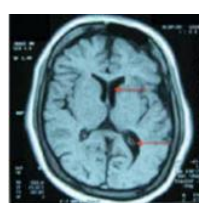

$\mathrm{CR}=21.02$, $\mathrm{PSNR}=31.43$ $\mathrm{MSE}=46.71$

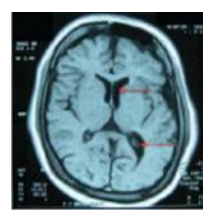

$\mathrm{CR}=17.25$

$\mathrm{PSNR}=32.43$

$\mathrm{MSE}=37.12$

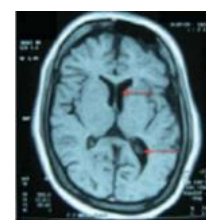

$\mathrm{CR}=15.11$, $\mathrm{PSNR}=33.0$ $\mathrm{MSE}=32.04$
10: Samples of the Reconstruct Value is Varied.

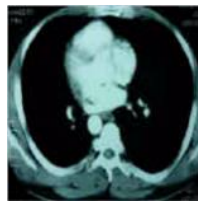

$\mathrm{CR}=46.09$, $\mathrm{PSNR}=30.71$ $\mathrm{MSE}=55.13$

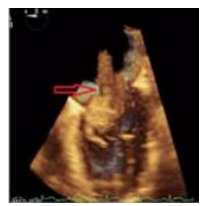

$\mathrm{CR}=38.23$, $\mathrm{PSNR}=31.02$ $\mathrm{MSE}=51.32$

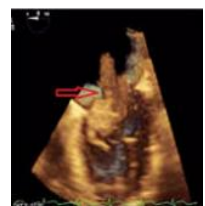

$\mathrm{CR}=31.04$, $\mathrm{PSNR}=32.02$ $\mathrm{MSE}=40.77$

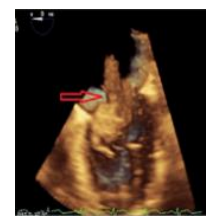

$\mathrm{CR}=$

25.76,PSNR=

33.20
$\mathrm{MSE}=31.06$

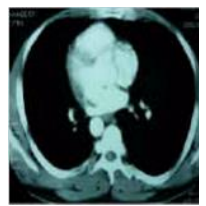

$\mathrm{CR}=40.83$, $\mathrm{PSNR}=31.40$ $\mathrm{MSE}=47.02$

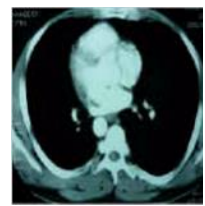

$\mathrm{CR}=38.40$, $\mathrm{PSNR}=32.00$ $\mathrm{MSE}=49.79$

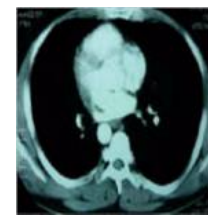

$\mathrm{CR}=32.61$, $\mathrm{PSNR}=33.00$ $\mathrm{MSE}=32.57$
Value Is Varied. Value is Varied.

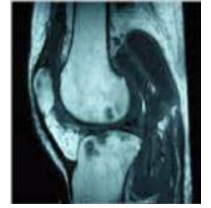

$\mathrm{CR}=36.95$ PSNR $=30.08$ $\mathrm{MSE}=63.71$
$\mathrm{MSE}=47.16$

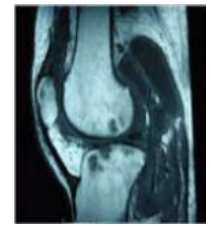

$\mathrm{CR}=25.63$ $\mathrm{PSNR}=32.13$ $\mathrm{MSE}=39.18$

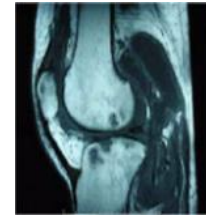

$\mathrm{CR}=19.15$, $\mathrm{PSNR}=33.08$ $\mathrm{MSE}=31.95$
Fig. 12: Samples of the Reconstructed MRI-Brain Knee Where the PSNR Value is Varied.

\section{Conclusion}

From the collected results and analysis of the proposed compression system based on the various evaluation parameters compression ratio (CR), peak signal to noise ratio (PSNR) and mean square error (MSE), we conclude that the using of hybrid DCT-DWT for image compression improves the CR, PSNR and MSE and gives better results than using only the DCT. They also show indicate that the proposed flipping block method improves the compression ratio when used with an adaptive RLE, which used to represent the zeros of vectors as a RUN to be compressed using an adaptive RLE. Using an image enhancement as a new flipping with an adaptive RLE improves the compression ratio of the reconstructed image and gives better results than does the traditional RLE method. Finally, the Compression ratio with shift coding decrease the time of encoding and decoding than using compression of image with Huffman coding. 


\section{Future works}

In this study, hybrid DCT-DWT, flipping block method, and using an image enhancement as a new flipping with an adaptive RLE improve the image compression ratio as we can see in table (1). These algorithms are developed and by performing some variation on basic ideas of these techniques to improve the CR and the performance of the system. Also we will try to use Arithmetic coding method to compare with the results of the above techniques.

\section{References}

[1] Kiran, Anita and Aman, "A Comparative Study of Image Compression Algorithms", International Journal of Research in Computer Science, Volume 2 Issue 52012.

[2] Anitha s, "Image Compression Using Discrete Cosine Transform \& Discrete Wavelet Transform", International Journal of Scientific \& Engineering Research Volume 2, Issue 8, August-2011.

[3] Anuja and Manisha, "DCT and DWT in Medical Image Compression", Volume-2, Issue-3, 2013.

[4] Er. Abhishek and Er. Deepti, "Image Compression Algorithms Using Dct", Journal of Engineering Research and Applications, Vol. 4, Issue 4(Version 1), April 2014.

[5] Shaymaa Dhyaa Aldeen Ahmed, "Image Compression using Adaptive Polynomial Transform", Master Thesis, 2018.

[6] Sai and Veerendra, "Comparison of Image Compression and Enhancement Techniques for Image Quality in Medical Images", Master Thesis Electrical Engineering February 2017.

[7] T. Karthikeyan 1 and C. Thirumoorthi, "A Hybrid Medical Image Compression Techniques for Lung Cancer", Indian Journal of Science and Technology, Vol 9, October 2016.

[8] Achinta and Dr. Lakshmi, "A COMPARATIVE STUDY ON LOSSY IMAGE COMPRESSION TECHNIQUES", International Journal of Current Trends in Engineering \& Research (IJCTER), Volume 2 Issue 6, June 2016.

[9] Nitu and Savita, "Comparative Analysis of Image Compression Using DCT and DWT Transforms", IJCSMC, Vol. 3, Issue. Seven, July 2014.

[10] Er. Ramandeep and Navneet, "Image Compression Using Discrete Cosine Transform \& Discrete Wavelet Transform", International Journal of Computing \& Business Research, 2012.

[11] Mr. Amit and Prof. Pravin, "A Survey on Medical Image Compression Using Hybrid Technique", International Journal of Computer Science and Mobile Computing, International Journal of Computer Science and Mobile Computing, Vol.4 Issue.2, February- 2015.

[12] Nitu and Savita, "Comparative Analysis of Image Compression Using DCT and DWT Transforms", IJCSMC, Vol. 3, Issue. Seven, July 2014.

[13] Qusay Kanaan Kadhi, "Image Compression Using Discrete Cosine Transform Method ", IJCSMC, Vol. 5, Issue. 9, September 2016.

[14] Sherin and Shaiju, "Review On Transform Based Medical Image Compression", 2012.

[15] Kiran, Anita and Aman, "A Comparative Study Of Image Compression Algorithms", International Journal of Research in Computer Science, Volume 2 Issue 52012. 